\title{
Authoring Step-Based ITS with ASTUS: An Interactive Event
}

\author{
Jean-François Lebeau, Luc Paquette, and André Mayers \\ Université de Sherbrooke, Québec, Canada \\ \{jean-francois. lebeau2, andre.mayers\}@usherbrooke.ca \\ http://astus.usherbrooke.ca
}

Step-based ITS have been proven successful for well-defined domains, particularly in well-defined tasks, but their success is mitigated by the amount of effort needed to build them. Typically, the main factor behind these efforts is the model of the task domain. Different approaches have been investigated to reduce these efforts: ModelTracing Tutors (e.g. Cognitive Tutors, Andes), Constraint-Based Tutors (e.g. SQLTutor, ASPIRE) and Example-Tracing Tutors (e.g. CTAT's, ASSISTment).

With ASTUS, we aim to offer to the ITS community support for the development of tutors for well-defined tasks in a wide range of task domains. In such context, building a framework based on a generative model of the task domain was deemed the most interesting approach because it appeared as the only one leading to comprehensive, flexible and re-usable pedagogical behaviors. For instance, the tutor is able not only to show next-step hints, but to generate them by instantiating domainindependent templates with domain-specific knowledge components.

ASTUS's knowledge representation system is based on manipulable knowledge components that encode tutored skills and "black-box" knowledge components that make operational the already mastered ones. Using an authoring language (prototyped with a Groovy-based Domain-Specific Language), the model can be encoded in coherent, easy-to-navigate files, similarly to typical source files. Tools for debugging and visualization are available at runtime.

Our first step with ASTUS was to reproduce tutors built with a comparable framework, for example we replicated a "scatter plot" tutor created with the Cognitive Tutors" "TDK" and we simultaneously developed a "multi-column subtraction" tutor using CTAT's Jess-based Cognitive Tutors and ASTUS. For the ASTUS-based tutor, we then reproduced the pedagogical behavior of the original tutor thanks to domainindependent pattern instead of domain-specific efforts.

As the ITS move from the labs to the classrooms, the next logical step may be to largely move the authoring efforts from highly specialized graduate students to domain experts (including teachers), but we are interested in investigating an intermediate step that consist in a comprehensive, flexible and usable framework for authors skilled in knowledge-based systems. We are aware that our approach, based on generative models, may be justified only in well-defined domains and that some ill-defined tasks, such as design-based ones, may be challenging at best. However, there is no such tool available for the ITS community that is explicitly designed to facilitate the experimentation of different pedagogical approaches.

Participants will interact with different tutors to observe the pedagogical behaviors offered by the framework and will be walked through authoring a change to a model. 